\title{
A hybrid content based image retrieval system using log-gabor filter banks
}

\author{
D. Madhavi ${ }^{1}$, Khwaja Muinuddin Chisti Mohammed ${ }^{2}$, N. Jyothi ${ }^{3}$, M. Ramesh Patnaik ${ }^{4}$ \\ ${ }^{1,2}$ Department of ECE, GIT, GITAM Deemed to be University, India \\ ${ }^{3}$ Department of EIE, GIT, GITAM Deemed to be University, India \\ ${ }^{4}$ Department of Instrument Technology, AUCE, Andhra University, India
}

\begin{tabular}{l} 
Article Info \\
\hline Article history: \\
Received Feb 14,2018 \\
Revised Sep 8, 2019 \\
Accepted Sep 21, 2018 \\
\hline Keywords: \\
Average Precision \\
Average Recall \\
Filtering techniques \\
Gabor Filter \\
Log-Gabor \\
\hline
\end{tabular}

\begin{abstract}
In this paper, a new efficient image retrieval system using sequential process of three stages with filtering technique for the feature selection is proposed. In the first stage the color features are extracted using color histogram method and in the second stage the texture features are obtained using logGabor filters and in the third stage shape features are extracted using shape descriptors using polygonal fitting algorithm. The proposed log-Gabor filter in the second stage has advantages of retrieving images over regular Gabor filter for texture. It provides better representation of the images. Experimental evaluation of the proposed system shows improved performance in retrieval as compared to other existing systems in terms of average precision and average recall.
\end{abstract}

Copyright $(2019$ Institute of Advanced Engineering and Science. All rights reserved.

\section{Corresponding Author:}

D. Madhavi,

Department of ECE,GIT,GITAM Deemed to be University,

Visakhapatnam, Andhra Pradesh, 530045-India.

Email: dmadhavi336@gmail.com

\section{INTRODUCTION}

Content based Image retrieval is a challenging task to recover exact similar type of images from the huge database. Many color, texture and shape feature extraction methods are available in literature. This paper proposes a new Query by image content system using a log-Gabor filter. Sequence of feature extraction in retrieval can be color, texture, shape or texture, shape color, and so on. It has been investigated that the best sequence that can be used to improve the retrieval efficiency is color features first, secondly texture and then the shape features [1]. The proposed image retrieval system is a hybrid method that is performed in three stages. In first stage images are retrieved based on color features similarity followed by texture and shape features in stage 2 and stage 3 respectively. Looking into methodology, in the first stage color features are extracted using color histogram and the similar L images to the given query image are obtained using similarity metric.

For texture feature extraction Gabor filters are generally used. However, the Gabor functions having certain drawbacks relating to their frequency response representation. In this paper, texture feature extraction using log-Gabor filters is proposed, which is the second stage of the proposed retrieval system. These filters are theoretically superior to Gabor filters as they can better represent the frequency properties of natural images. The $\mathrm{L}$ images that are obtained from stage 1 are given as the input data set for stage 2 . The most similar $\mathrm{M}$ images to the given query image are obtained from this stage 2 . In stage 3 , image retrieval is based on shape feature extraction using curve polygonal fitting algorithm. The best $\mathrm{N}$ images to the query image are obtained from this stage.

The main important features in image retrieval is texture. Different methods have been published by many researchers in the area of image retrieval, especially they differ in the process of extracting the texture 
feature. In [2] image retrieval process using a set of Gabor filters and the feature vector is constituted using mean and standard deviation. Retrieval system using Gabor and Curvelet transform for retrieval of three traditional paintings proposed in [3]. However it has not been used for combination of feature extraction. In [4] comparision of different feature extraction techniques for CBIR using color texture and shape are presented.Gabor filters are used for gender recognition from face images in [5]. To improve the retrieval efficiency of Content Based Image Retrieval system color and texture features are combined [6] and three moments of the color distribution are evaluated and texture features are extracted using Gabor filters. The similarity is measured using Canberra distance.

Gabor filters suggested [7] for the representation of color texture using opponent and unichrome features. In [8] image retrieval system using color, texture and shape features extraction with support vector machine. Gabor filters for vehicle detection proposd in [9], the derived filters are evaluated using fitness function based on support vector machines.Gabor filters triggered with different frequency, orientation and scale [10] with minimization of energy computation for texture feature selection. In [11] optimized Gabor filters are proposed for detection of defect in surface. Genetic algorithm optimized gray and color Gabor filters in CBIR system design are considered in [12-13]. These research outputs shows that use of Gabor filter for the texture extraction has most impact of reterival performance. However the Gabor filters are not having zero mean and hence the frequency spectrum is not covered uniformly. So in this paper a log-Gabor filter is considered for the texture feature extraction.

Literature also shows that log-Gabor filters have the advantage over the traditional Gabor in the area of texture description and analysis. In [14] log-Gabor filters are used for automatic vehicle detection. Gabor and $\log$ Gabor filters approach for texture feature attraction and the texture metrics are measured using Kullback-Leibler and the Jensen-Shannon divergence [15]. Shail Kumari Shah et.al [16] proposed recognition of three facial emotions-happy, neutral, surprise using Gabor, log-Gabor and principal component analysis (PCA). The features of the face are extracted using Gabor and log-Gabor filters and the dimensionality of the feature vector obtained is reduced by PCA. However the images of low resolution and different rotations of head positions are not identified properly. Log-Gabor filters and support vector machines are used for vehicle verification [17] on the road and their performance is compared with the performance using traditional Gabor filters. In [18] Image retrieval system using color and shape features with ESPRIT algorithm and Gabor filters are proposed. Gabor filters for different orientations and angles are applied for image enhancement of finger print [19].

So, to take the advantage of the log-Gabor filter, in this paper we proposed log-Gabor filter bank for the image retrieval in the second stage. The design of filter bank is based on different scale and orientation parameters is discussed in the next section. This paper is organized as follows. In section 2 the functional block diagram of the proposed image retrieval system is presented. In section 3 feature extraction of all three stages are explained. Experimental analysis and results are shown in section 4. Conclusions are presented in section 5 .

\section{PROPOSED METHOD}

The functional block diagram of the proposed sequential three stage-hybrid image retrieval system is shown in Figure 1. K database images are considered as input set. In stage 1, the best L relevant images to the query image are obtained using HSV color space with color histogram. These L images are given as input data set for stage 2. In this stage, a log-Gabor filter is used for texture feature extraction and best $\mathrm{M}$ images are identified using minimum energy feature. These $M$ database images are selected as a data set for stage 3 . In this stage, image retrieval is considered using shape feature extraction with polygonal curve fitting algorithm. The best $\mathrm{N}$ images are extracted from this stage which are the best possible relevant images to the given query image. We considered the relation in number of images that are extracted in each stage of the proposed retrieval system as $\mathrm{K}>\mathrm{L}>\mathrm{M}>\mathrm{N}$.

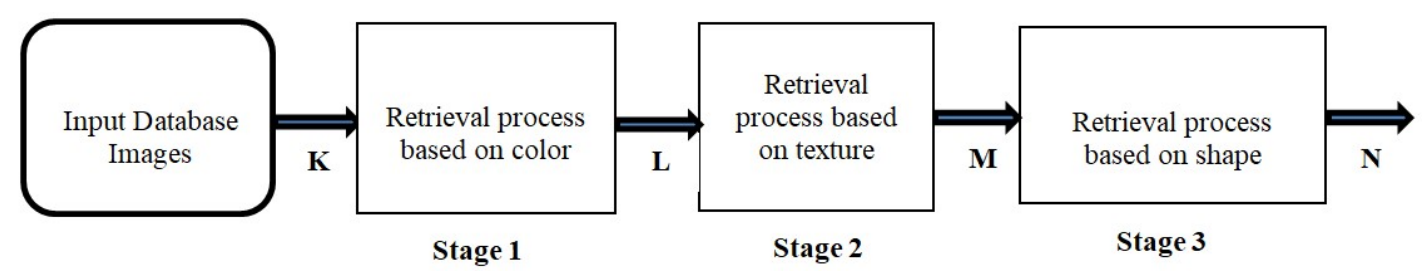

Figure 1. Functional blocks of three stage - Hybrid system for image retrieval system 


\section{FEATURE EXTRACTION METHODS}

Features are extracted based on the color, texture and shape. In this section, we discuss the process of feature extraction and the similarity measure used at each stage. The computational units in these stages are shown in Figure 2. The extracted ' $i$ ' images from one stage are passed as input to the next successive stages where ' $\mathrm{i}$ ' takes values as $\mathrm{K}, \mathrm{L}$ and $\mathrm{M}$ respectively at each stage. The following sub sections gives details of extraction of these ' $i$ ' images at each stage namely color, texture and shape.

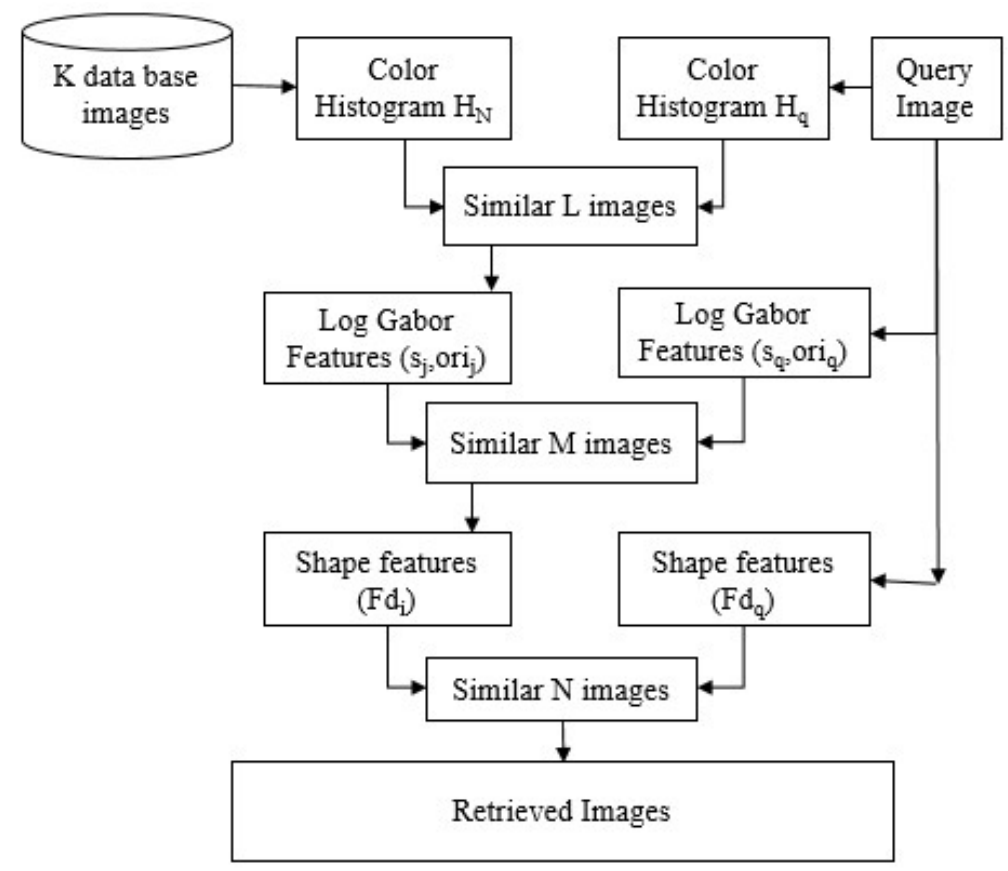

Figure 2. Computational units of the proposed retrieval system

\subsection{Retrieval process based on color features}

Color features extraction is an important part in human recognition system. It is an easiest and widely used method in CBIR. This feature is not variant with size and rotation of the picture. Different methods used for color feature extraction are color histogram, correlogram. In this paper, the color information is extracted from the large data set using color histogram. The color content extracted using HSV color space and these values are quantized [20].

HSV histogram of $n$ bins is used for the $K$ input data set and query image for feature extraction [1]. The similarity between these is obtained by the distance measurement. The $\mathrm{L}$ images which are having highest distance metric are considered and other are ignored, these L images are passed as input dataset to the texture feature extraction stage. be given by:

If $\mathrm{x}$ and $\mathrm{y}$ are two histograms with size ' $\mathrm{n}$ ' then the distance $D$ between the histograms $\mathrm{x}$ and y can

$$
D=\frac{\sum_{j=1}^{n} \min (x[j], y[j])}{\min (|x|,|y|)}
$$

Where $|x|$ and $|y|$ are histogram magnitudes. This distance measurement is arranged in increasing order and first L images are having best similar images to query image.

\subsection{Rretrieval process based on log-gabor filter}

Gabor filters are generally used for extracting texture features of images. However the high DC component of these filters requires more number of filters for spectrum coverage. The frequency response of these filters also symmetrically distributed at center frequency, which causes redundant data at low frequencies. To overcome these limitations log Gabor filters are considered for texture feature extraction. The symmetry of the log axis facilitates uniform coverage of the frequency spectrum and reduces the redundancy at low frequencies. The log-Gabor filters having no DC component which allows an increase in the 
bandwidth, and hence fewer filters are required to cover the same spectrum as compared to original Gabor filters.

Filter over comes angular wrap around problem, for which it consider cosine and sine difference as shown in (2) and (3).

$$
\begin{aligned}
& D s=\sin \theta * \cos \emptyset-\cos \theta * \sin \emptyset \\
& D c=\cos \theta * \cos \emptyset+\sin \theta * \sin \emptyset
\end{aligned}
$$

Where $\theta=\tan ^{-1}\left(\frac{-y}{x}\right)$ with $\mathrm{x}$ and $\mathrm{y}$ as allowed size of the filter, radius $=\sqrt{x^{2}+y^{2}}$, orientation $\emptyset=0$ to $(2 \mathrm{n}-1) \pi / \mathrm{n}$, with $\mathrm{n}$ as number of orientations.

$$
A d=\left|\tan ^{-1}\left(\frac{D s}{D c}\right)\right|
$$

The log Gabor filter introduced by Field, and its frequency domain representation is given by:

$$
G(w)=e^{\left(\frac{-\left(\log \left(w / w_{0}\right)\right)^{2}}{2(\log (\sigma))^{2}}\right)}
$$

Here $w_{0}=1 / \lambda$, where ' $\lambda$ ' changes with the specific scale, here the resultant value of the ' $\lambda$ ' selected as wavelength $* \mathrm{~s}$; where $\mathrm{s}$ is a scaling factor between different filters.

Where $\sigma$ is standard deviation of the angular Gaussian function and $w_{0}$ is its center frequency. The bandwidth of the filter is effected by the variable $\sigma$.

By considering not only the particular frequency but also the orientation factor, the two dimensional log- Gabor filter is given by (6).

$$
G(f, \theta)=G(w) \cdot e^{\left(\frac{-A d^{2}}{2 \sigma_{\varnothing}^{2}}\right)}
$$

Here $\sigma_{\varnothing}=\frac{\pi}{n * k}, \mathrm{k}=$ interval between filter orientations $/ \sigma$

The texture features are obtained by taking a log-Gabor filter which is specified by (5) for different scales and orientations and are shown in Figure 3. A total of 12 filter banks are obtained by considering three scales and four orentations. The energy functions are computed by convolving these filters with the query and the each data set images. These energy functions are used as feature set for matching the query image with the data set. The similarity between the energy features are computed to choose the best matched images. The best $\mathrm{M}$ images that with most relavent energies to the query image are obtained from this stage. 


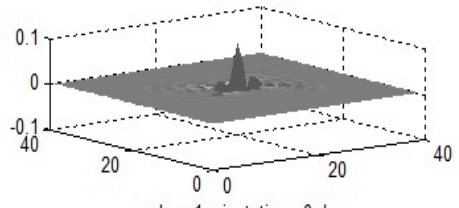

scale $=1$, orientation $=0 \mathrm{deg}$
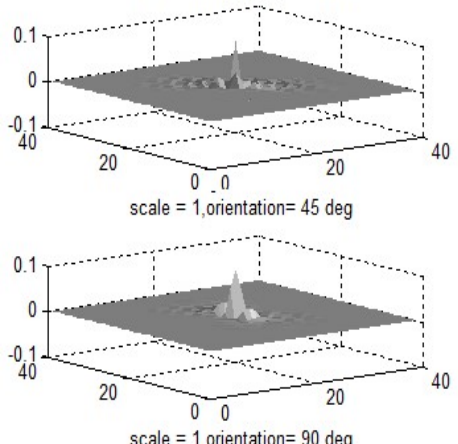

$=1$, orientation $=90 \mathrm{deg}$

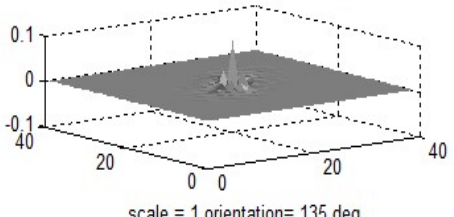

scale $=1$, orientation $=135 \mathrm{deg}$
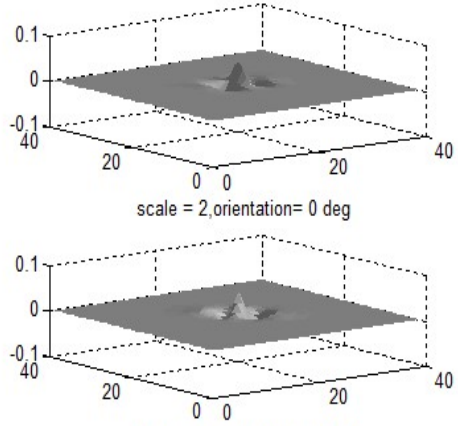

scale $=2$, orientation $=45 \mathrm{deg}$

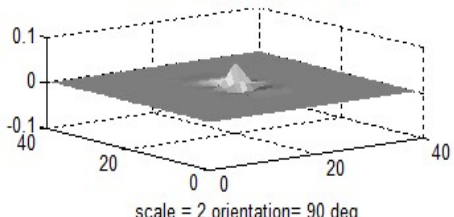

cale $=2$, orientation $=90 \mathrm{deg}$

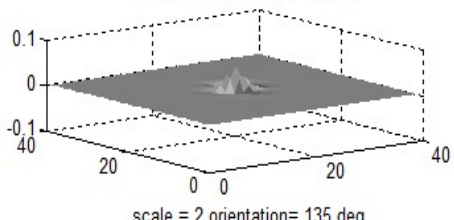

scale $=2$, orientation $=135 \mathrm{deg}$
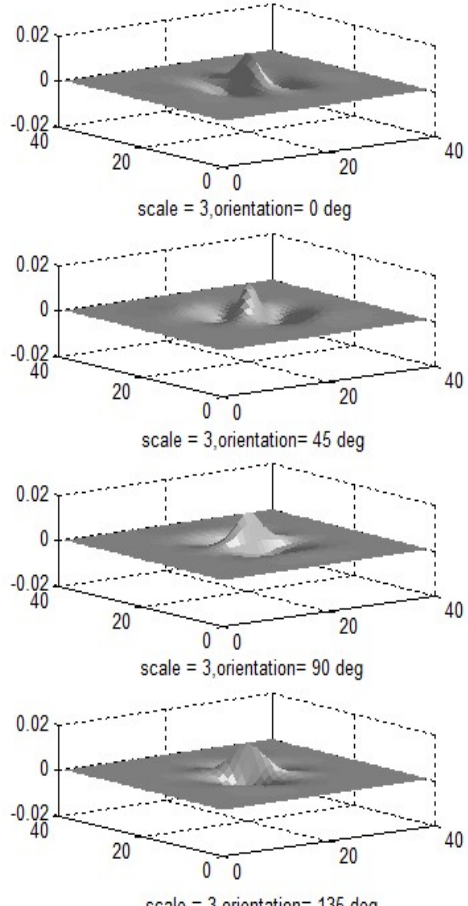

scale $=3$, orientation $=135 \mathrm{deg}$

Figure 3. Bank of log- Gabor filters with scale $=3$ and orientation=4 (A total of 12 filters)

\subsection{Retrieval process based on shape features}

The best $\mathrm{M}$ images that are obtained from the previous stage are considered as the input database for this third stage. Here the Fourier representation of the shape signature functions are computed and the outer boundary shape is developed using polygon fitting algorithm [20]. The minimal distance between the pixels is considered to be one unit. The magnitude of shape descriptors for query image and all the input data set of stage 3 are computed and Euclidean distance metric is calculated between query image and all the input data set using (7).

$$
d=\sqrt{\sum_{i=1}^{M}\left|f_{q}^{i}-f_{d}^{i}\right|^{2}}
$$

Where $\mathrm{f}_{\mathrm{q}}^{\mathrm{i}}$ the characteristic vector of is required image and $\mathrm{f}_{\mathrm{d}}^{\mathrm{i}}$ is the characteristic vectors of all input data set images of stage 3 .

\section{RESULTS AND ANALYSIS}

The experimental analysis of the proposed log-Gabor filter based image retrieval system is analyzed by using Wang database [21] by taking 1000 images of 10 classes. An elephant image in the dataset is considered as query image and is shown in Figure 4. The values of L, M and $N$ are taken as 8,6 and 3 respectively.The best 8 images that are relevant to query image are obtained in stage 1 and is given as input set for stage 2. From stage 2, six best possible images are obtained and it is applied as input data set for stage 3. Finally from stage 3 best 3 images are extracted. The retrieval results of each stage are shown in Figure 5, 6, 7

The efficiency of the proposed retrieval scheme is tested by the performance metrics Average Precision (A.P) and Average Recall (A.R) which are defined as:

A.P $=\frac{\text { No. of relevant images retrieved }}{\text { No. of images retrieved }}$

$A . R=\frac{\text { No.of relevant images retrieved }}{\text { No.of relevant images in the data set }}$ 


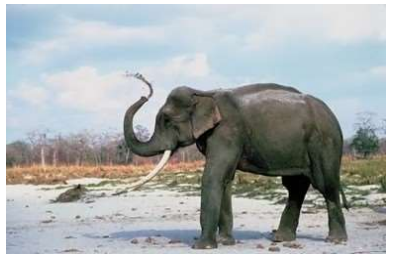

Figure 4. Query image
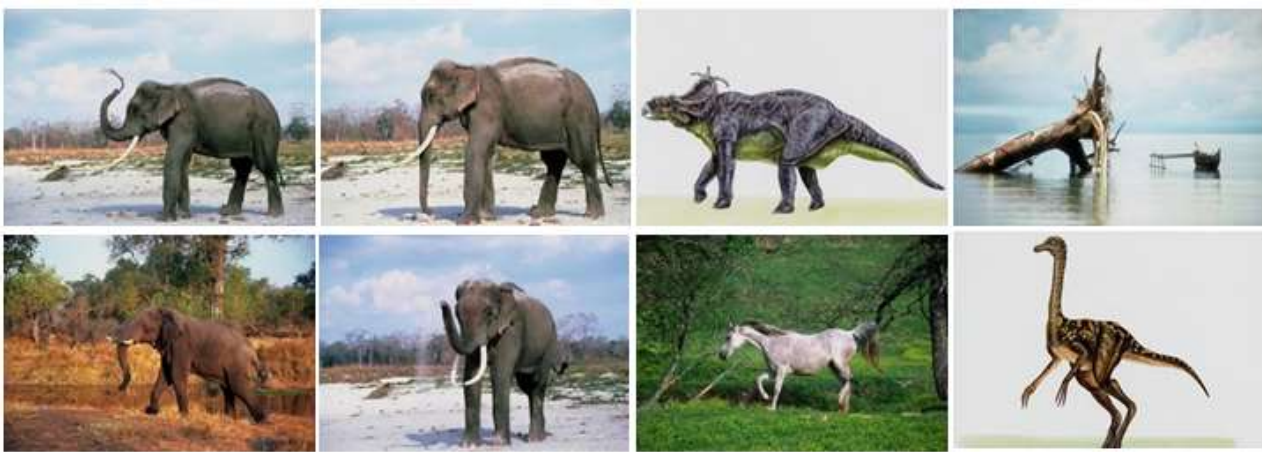

Figure 5. Stage 1 retrieved images with $\mathrm{L}=8$
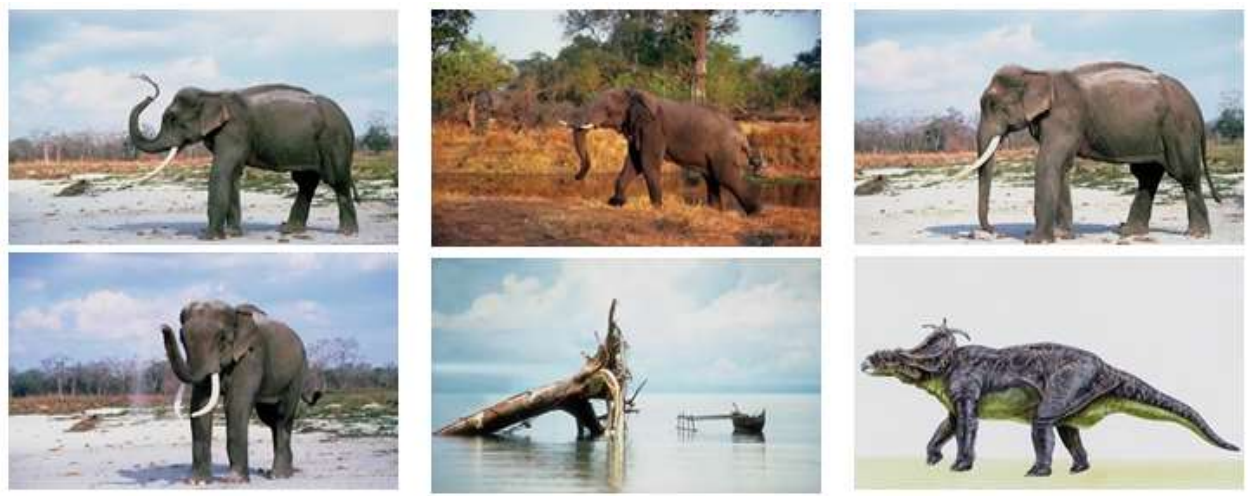

Figure 6. Stage 2 retrieved images with $M=6$
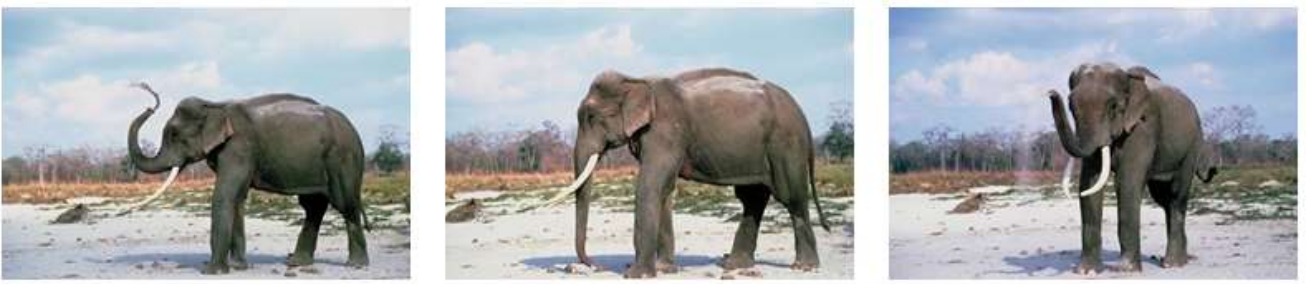

Figure 7. Stage 3 retrieved images with $\mathrm{N}=3$

By using retrieval outcomes of each of these images, the performance of the proposed log- Gabor filter based CBIR system is compared with the Nishant model [1] and Chuen model [22]. The Average Precision and Average Recall are compared by varying the number of retrieved images from 20 to 100 and are shown in Figure 8 and Figure 9. It is observed that the proposed log-Gabor filter based system shows improved average Precision and average Recall compared to other two models. 


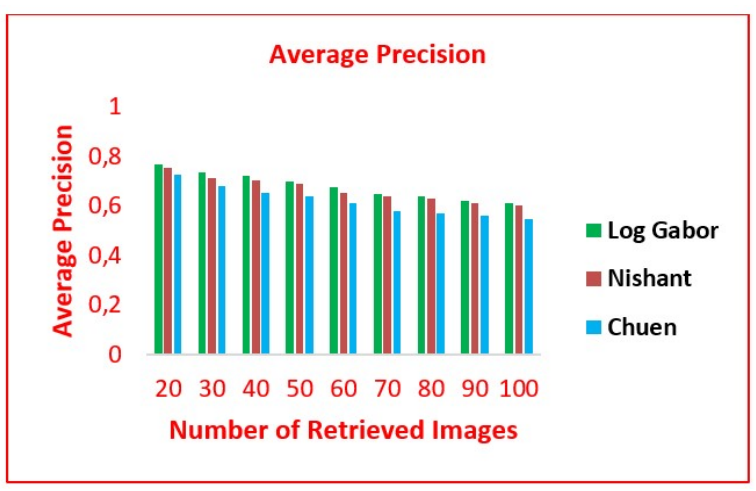

Figure 8. Comparison of average precision of the proposed log-gabor filter based on using of CBIR system and Nishant Shrivastava model, and Chuen model

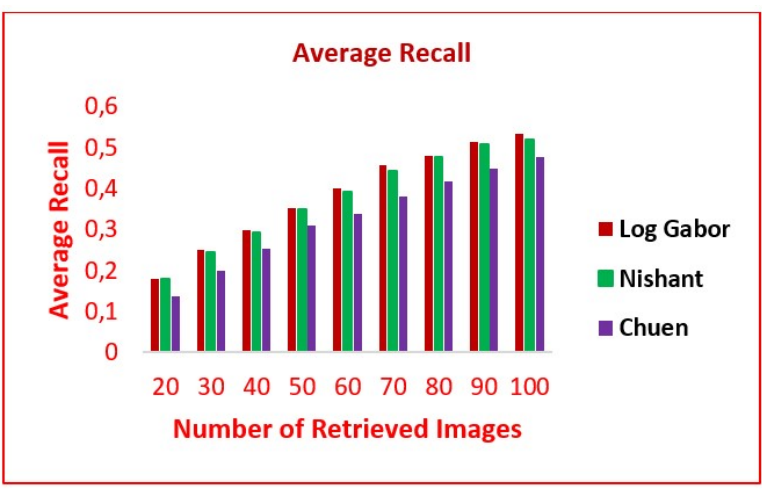

Figure 9. Comparison of average recall of the proposed log-gabor filter based on using of CBIR and

Nishant Shrivastava model, and Chuen model

\section{CONCLUSION}

Content based image retrieval is a challenging task for obtaining similar images from a large database. In this paper a sequential process of color, texture and shape feature analysis of a CBIR system has been proposed. The proposed system makes use of the advantages of log- Gabor filter over the traditional Gabor filter, which has shown a visible difference in terms of retrieval performance metirs. The effectiveness of the proposed system verified using performance metrics of average precision and average recall. The experiments conducted in this paper confirm that the proposed CBIR system using log-Gabor filters for texture feature extraction significantly outperforms the original Gabor filter for content based image retrieval.

\section{REFERENCES}

[1] N. Shrivastava and V.Tyagi., "An Efficient Technique for Retrieval of Color Images in Large Databases," Computers and Electrical Engineering, vol.46, pp. 314-327, August 2015.

[2] Manjunath, B.S., Ma, W.Y., "Texture Features for Browsing and Retrieval of Image Data," IEEE Transactions on Pattern Analysis and Machine Intelligence, vol.18, pp. 837-842, 1996.

[3] Pooja Manjarekar, Priya Saxena., "Traditional Indian Painting Retrieval System Based on the Fusion of Curvelet and Gabor Transform," International Journal of Engineering Sciences \& Research Technology, vol. 5, pp.579-584, Dec. 2016.

[4] Mayank Jain and Divakar Singh., "A Survey on CBIR on the Basis of Different Feature Descriptor," British Journal of Mathematics \& Computer Science, vol.14, pp. 1-13, Feb 2016.

[5] M. J. Barani, et al., "Implementation of Gabor Filters Combined with Binary Features for Gender Recognition," International Journal of Electrical and Computer Engineering (IJECE), vol.4, pp.108-115, Feb 2014.

[6] S. Mangijao Singh, K. Hemachandran, "Content-Based Image Retrieval using Color Moment and Gabor Texture Feature," International Journal of Computer Science Issues, vol. 9, Sept 2012.

[7] Amit Jain and Glenn Healey., "A Multiscale Representation Including Opponent Color Features for Texture Recognition," IEEE Transactions on Image Processing, vol. 7, Jan 1998.

[8] T. G. Ngo, et al., "Image Retrieval with Relevance Feedback using SVM Active Learning," International Journal of Electrical and Computer Engineering (IJECE), vol.6, pp.3238-3246, Dec 2016.

[9] Zehang Sun, George Bebis, and Ronald Miller., "On-road Vehicle Detection Using Evolutionary Gabor Filter Optimization," IEEE Transactions on Intelligent Transportation Systems, vol. 6, pp.125-137, Jun 2005.

[10] D. M. Tsai, et al., "Defect Detection in Colored Texture Surfaces using Gabor Filters," The Imaging Science Journal, vol. 53, pp.27-37, 2005.

[11] K. M .Chisti, K. S. Srinivas, and G. Prasad, "2D Gabor Filter for Surface Defect Detection Using GA and PSO Optimization Techniques," AMSE Journals, vol. 58, pp.67-83, 2015.

[12] D. Madhavi and M. R. Patnaik., "Image Retrieval Using GA Optimized Gabor Filter," Indian Journal of Science and Technology, vol. 9, pp.1-11, 2016.

[13] D. Madhavi and M. R. Patnaik., "Image Retrieval Based on Tuned Color Gabor Filter Using Genetic Algorithm," International Journal of Applied Engineering Research, vol.12, pp.5031-5039, 2017.

[14] Jon Arrospide and Luis Salgado., "Log-Gabor Filters for Image-Based Vehicle Verification," IEEE Transactions on Image Processing, vol. 22, pp.2286-2295, June 2013.

[15] Rodrigo Nava, Boris Escalante-Ramirez, Gabriel Cristobal., "Progress in Pattern Recognition, Image Analysis, Computer Vision, and Applications," 17th Iberoamerican Congress, CIARP 2012, 2012, pp 414-421.

[16] Shail Kumari Shah, Vineet Khanna., "Facial Expression Recognition for Color Images using Gabor, Log Gabor Filters and PCA," International Journal of Computer Applications, vol. 113, pp.42-46, Mar 2015. 
[17] S. Chitradevi, Mr. G. Rajasekaran., "On Road Vehicle Verification Using Log- Gabor Filters and Support Vector Machines," International Journal of Engineering Science and Computing, vol.3, pp. 481-485, May 2014.

[18] C.Youness, EI A. Khalid, O. Mohammed, A.Brahim., "New Method of Content Based Image Retrieval based on 2D ESPRIT Method and the Gabor Filters," TELKOMNIKA Indonesian Journal of Electrical Engineering, vol.15, pp. 313-320, August 2015.

[19] P. Pakutharivu, M.V. Srinath., "Analysis of Fingerprint Image Enhancement Using Gabor Filtering with Different Orientation Field Values," Indonesian Journal of Electrical Engineering and Computer Science, vol. 5, pp. 427432, Feb.2017.

[20] R.Gonzales, and R. E. Woods, “Digital Image Processing,” 2nd Edition, New Jersey Prentice Hall, 2002.

[21] Wang Database : http://wang.ist.psu.edu/

[22] Chuen-Horng Lin, et al., "A Smart Content-based Image Retrieval system based on Color and Texture feature," Image and Vision Computing, vol.27, pp.658-665, May 2009. 